\title{
HERRAMIENTAS DE AYUDA AL DIAGNÓSTICO DE LA NEUMONÍA PENUMONÍA DIAGNOSIS AID TOOLS
}

\author{
Ing(c). Kevin Andrée Sarmiento Velasco*, Ing(c). Oscar Andrés Bolaños Tapia* \\ PhD. Diego Ferney Gómez Cajas*, PhD. Álvaro David Orjuela Cañón**
}

\author{
Universidad Antonio Nariño, Facultad de Ingeniería Biomédica, Electrónica y \\ Mecatrónica, Grupo de Investigación GIBIO \\ *Sede El Tablazo, Popayán, Colombia. \\ ** Sede Circunvalar, Bogotá, Colombia. \\ E-mail: \{skevin, obolanos, dfgomezc, alvorjuela\}@uan.edu.co.
}

\begin{abstract}
Resumen: Este artículo realiza una revisión bibliográfica de diversos estudios presentes en la literatura que abordan soluciones tendientes a brindar ayudas al diagnóstico de la neumonía. Mediante el uso de nuevas tecnologías y nuevos procedimientos, se han abierto un gran abanico de posibilidades no solo al personal médico, sino también al público en general. Estos adelantos, buscan brindar cada vez una mayor y mejor información sobre esta letal enfermedad a pacientes y personal médico, así como grandes posibilidades de un diagnóstico temprano. Por lo tanto, el propósito de este estudio, es dar a conocer las diferentes aproximaciones planteadas en la literatura, al respecto de las tecnologías creadas para ayudar al diagnóstico de la neumonía.
\end{abstract}

Palabras clave: Neumonía, herramientas de ayuda al diagnóstico, reconocimiento de patrones, nuevas tecnologías.

\begin{abstract}
This article makes a bibliographical review of several studies present in the literature that address solutions tending to provide aid to the diagnosis of pneumonia. Using new technologies and new procedures, a wide range of possibilities have appeared not only to medical personnel, but also to the public. These advances, seek to provide more and better information about this lethal disease to patients and medical personnel, as well as great possibilities of an early diagnosis. Therefore, the purpose of this study is to make known the different approaches proposed in the literature, regarding the technologies created to help diagnose pneumonia.
\end{abstract}

Keywords: Pneumonia, diagnostic tools, pattern recognition, new technologies.

\section{INTRODUCCIÓN}

Para empezar, y de acuerdo a diferentes estudios presentes en la literatura; a continuación, se realizará una introducción estadística de los diversos tipos de enfermedades más letales. En primer término, se ha podido evidenciar un alto índice de morbilidad y mortalidad debido a Enfermedades No Transmisibles (ENT) en todo el planeta. De tal forma que se detectan aproximadamente 40 millones de muertes cada año, que equivalen a un $70 \%$ de todas las muertes que se presentan en todo el mundo (OMS, 2017). Concretamente en América, de acuerdo a la Organización Panamericana de la Salud (OPS), en el año 2016 las ENT fueron responsables de aproximadamente 4.8 millones de muertes (PAHO, 2016). Por otra parte, los cuatro tipos de ENT más frecuentes a nivel global son: las enfermedades cardiovasculares, el cáncer, la diabetes y las 
enfermedades respiratorias crónicas (OMS, 2017). Estas mismas, son responsables del $15 \%$ de las muertes en personas entre los 30 y los 70 años, conforme al último informe disponible de la Organización Mundial de la Salud (OMS) (WHO, 2017).

Dentro de las ENT, las Enfermedades Respiratorias Crónicas (ERC), son causantes de aproximadamente 3.9 millones las muertes a nivel global; observándose una mayor incidencia de éstas en países de bajos y medianos ingresos (OMS, 2017;¡Error! No se encuentra el origen de la referencia. Beran et al, 2015). En América, las ERC corresponden al $8.1 \%$ de las ENT, equivalentes a cerca de 400 mil muertes (PAHO, 2016). Adicionalmente, en Colombia según datos del Ministerio de Salud y Protección Social del año 2016, se evidenció que entre los años 2005 y 2013, las enfermedades respiratorias crónicas fueron causantes de aproximadamente 106 mil defunciones (Ministerio de Salud y Protección Social, 2016).

De otra parte, en cuanto a las Enfermedades Transmisibles (ET), por cada 100.000 habitantes, hay 75.5 fallecidos en el continente americano (PAHO, 2014). Pero, dentro de las ET, merecen especial atención las Enfermedades Respiratorias Agudas (ERA), pues éstas constituyen la principal causa de morbilidad y mortalidad en niños menores de cinco años en el mundo (Pio y Leowski, 1984). Concretamente en Colombia, entre los años 2005 y 2014, se produjeron 66.346 muertes por este tipo de enfermedades, equivalente a $49,46 \%$ de todas las muertes por Enfermedades Transmisibles (Ministerio de Salud y Protección Social, 2016).

Dentro de las ET, las Infecciones Respiratorias Agudas (IRA), son la principal causa de utilización de los servicios de salud en los países en desarrollo, incluida Colombia (Pio y Leowski, 1984).

Finalmente, la neumonía, categorizada dentro de las Infecciones Respiratorias Agudas, fue responsable en países en vía de desarrollo, de una incidencia anual estimada de 151 millones de nuevos casos de NAC (Neumonía Adquirida en Comunidad, ver Sección 2.1) en niños pequeños; de los cuales 10 a 20 millones son graves (Cruz et al, 2012). A nivel mundial, la neumonía fue la responsable de aproximadamente 920.136 muertes en niños menores a 5 años (equivalente al $15 \%$ de todas las defunciones) (OMS, 2016).
Por otra parte, aproximadamente 1 de cada 5 muertes de niños en el mundo, se producen por la neumonía, y en América Latina, aproximadamente el $14 \%$ de las muertes de niños son debidas a esta enfermedad; siendo la que más muertes provoca en éstos, incluso, más que el sida, el paludismo y el sarampión combinados (UNICEF, 2007).

Dado lo anterior, este artículo muestra los avances y diversos enfoques que, desde la tecnología se han concentrado en apoyar el diagnóstico temprano de la enfermedad; dado que con éste se podría obtener una reducción significativa en la tasa de mortalidad, especialmente en niños y personas en condiciones de alta vulnerabilidad (Mayhua-López et al, 2009). A continuación, se realizará una descripción general de la enfermedad, así como sus tipos y métodos tradicionales de diagnóstico.

\section{LA NEUMONÍA}

La neumonía es una Infección Respiratoria Aguda que afecta a los pulmones. En ellos se encuentran unos diminutos sacos llamados alveolos, que en personas sanas se llenan de aire al respirar, mientras que en personas enfermas (con neumonía), se llenan de líquido. Lo anterior ocasiona una respiración dolorosa, limitando la absorción de oxígeno en el cuerpo, y pudiendo provocar incluso la muerte (Cook et al, 1998). A continuación, se realiza una clasificación de la enfermedad, para detallar sus causas y efectos en el cuerpo humano.

\subsection{Clasificación.}

Antes de aplicar un procedimiento de atención a un paciente que padezca neumonía, es muy importante detectar el tipo concreto de neumonía que padece; pues así se puede aplicar un tratamiento eficaz y con menor riesgo (Fernández y Garro, 2015). En este sentido, se pueden realizar diversas clasificaciones (García y Aspa, 2005):

Dependiendo del parámetro comparado:

- Neumonía Adquirida en Comunidad (NAC). También llamada Neumonía ExtraHospitalaria $(\mathrm{NEH})$; se presenta en pacientes que no han sido internados en un hospital, o que no hayan ingresado en un centro médico con anterioridad a dos semanas en que se presentan los síntomas de la enfermedad. También se considera presente la NAC, en aquellos pacientes cuyos síntomas aparecen dentro de las primeras 24-48 horas de haber sido hospitalizados (INFAC, 2016). 
- Neumonía adquirida en los centros de atención médica. Conocida como Neumonía IntraHospitalaria (NIH); se presenta luego de pasadas 48 horas de ingreso en una entidad hospitalaria. La NIH, también se relaciona con otra clase de neumonía: la Neumonía Asociada a la Ventilación $(N A V)$, que se presenta en pacientes hospitalizados que requieren ventilación mecánica y el uso de una vía respiratoria artificial (Luna et al, 2005). De acuerdo al tiempo de aparición de la NIH (después de ingresar al hospital), se clasifica en: NIH temprana (menor a 7 días), o NIH tardía (después de 8 días), causada por agentes perjudiciales que colonizan la oro faringe de los pacientes (Luna et al, 2005).

De otra parte, también existen otras clasificaciones en función del agente que detona la aparición de la enfermedad:

- Viral. En este caso se produce una inflamación o hinchazón de los pulmones causada por un germen o virus, provocando dolor al respirar (MedlinePlus, 2017c). Este tipo de neumonía, se presenta con mayor frecuencia en niños menores, $\mathrm{y}$ adultos de edad avanzada; dado que, en ambos casos, el cuerpo no cuenta con las defensas necesarias, haciendo que dicha población padezca una mayor dificultad para combatir el virus (Fernández y Garro, 2015).

- Bacteriana. Cuando la infección que se presenta es debida a bacterias, los pulmones se inflaman (Allina, 2015). Este tipo de neumonía se presenta con menor frecuencia, y su tratamiento se basa en un diagnóstico bien fundamentado (Luna et $a l$, 2005). El agente etiológico principal que causa este tipo de neumonías, es el Streptococcus Pneumoniae, el cual presenta distintas variaciones que responden de manera diferente al tratamiento con antibióticos (Luna et al, 2005).

$\mathrm{Si}$ bien diferenciar el agente que denota la neumonía es clave para llevar a cabo un tratamiento eficiente, los avances tecnológicos tradicionalmente utilizados en las diferentes pruebas diagnósticas, no suelen permiten una diferenciación eficaz con respecto al tipo de neumonía que presenta el paciente; pues muchos de los síntomas suelen solaparse y resulta difícil diferenciar con certeza, entre neumonía viral y neumonía bacteriana ¡Error! No se encuentra el origen de la referencia.Finalmente, existen otras clasificaciones más diversas en función de los cuadros clínicos y/o pronósticos médicos, pero éstas no se detallarán en este artículo ¡Error! No se encuentra el origen de la referencia.Álvarez et al. 2017).

\subsection{Problemática asociada a la enfermedad.}

Existen diversos problemas que giran en torno al padecimiento de la neumonía, los cuales afectan en mayor medida a la población infantil y de la tercera edad; aumentando dicha incidencia, en países en vía de desarrollo, en regiones apartadas y de bajos recursos (Beran et al, 2015).

Problemática económica: La neumonía implica altos costos para los sistemas de salud, tal como lo demuestran diversos estudios: en Colombia, el costo total de tratamiento asociado a la Neumonía Adquirida en la Comunidad (NAC) es de aproximadamente Col\$12.000.000 (Calderón y Dennis, 2014), especialmente cuando los pacientes deben ser hospitalizados, pues de ser así, la atención hospitalaria consume más del $90 \%$ de los costos que genera esta enfermedad ¡Error! No se encuentra el origen de la referencia. De otra parte, los costos en las instituciones colombianas que involucran el tratamiento de neumonías presuntamente bacterianas son de aproximadamente USD \$611.50, mientras que las virales corresponden a USD \$ 472.20, según estudios realizados en varias ciudades colombianas, en niños menores a 2 años (Guzmán y de la Hoz, 2005).

Problemática social (Ordoñez et al, 2015):

De otra parte, los determinantes sociales de riesgo son aquellos factores que aumentan la probabilidad de contraer la enfermedad. Dentro de éstos, se destacan:

La edad: la neumonía de origen viral, presenta aproximadamente una mayor probabilidad de contraer la neumonía en niños menores a dos años, debido a su inmadurez inmunológica. También, las personas de la tercera edad, son más propensas a adquirir la enfermedad.

El género: en algunos estudios se menciona el predominio de la enfermedad en los hombres, debido a su mayor sensibilidad a cambios y/o alteraciones en el medio ambiente.

Lactancia materna: el uso de sustitutos a la leche materna puede generar diarreas, tos y catarros que predisponen la aparición de la neumonía.

Condiciones de hacinamiento: la falta de higiene en cocinas, puede ser un factor de riesgo para la adquisición de la neumonía. La presencia de 3 o más niños menores a 5 años, se asocia al aumento de la probabilidad de muertes por neumonía. 
Desnutrición: la mal nutrición, es una causa subyacente de las muertes en menores de 5 años por infecciones como la neumonía.

Enfermedades asociadas: diversas alteraciones respiratorias afectan el desarrollo inmunológico en lactantes, causando una disminución de su respuesta defensiva frente a infecciones como la neumonía.

Situación económica: la incidencia anual de la neumonía en países desarrollados es de 3 a 4\%, mientras que, en países en vía de desarrollo, dicha incidencia puede llegar al $20 \%$ (Alvis et al, 2014).

Nivel de instrucción de la madre: el mayor riesgo para los niños, se presenta en madres con bajos niveles de instrucción.

\section{DIAGNÓSTICO TRADICIONAL}

Comúnmente, la neumonía se detecta a partir de un examen físico evaluando distintos sonidos. Éstos se crean a partir de una inhalación y exhalación en los pulmones, que luego son captados por un estetoscopio que utiliza el médico para analizarlos.

Por otra parte, para dar un diagnóstico más preciso se pueden realizar una o más de las siguientes pruebas: Radiografía de tórax, pruebas de sangre, medición de oxígeno en sangre, prueba de esputo (si el paciente está hospitalizado), tomografía computarizada de tórax, cultivo de líquido pleural, oximetría de pulso y broncoscopia ¡Error! No se encuentra el origen de la referencia.:

A continuación, se explican algunas de ellas: Auscultación: por medio de un estetoscopio, se logran escuchar de una mejor manera los sonidos pulmonares. Durante esta prueba el médico puede determinar si el paciente presenta sonidos disminuidos en la respiración o ruidos anormales en los pulmones, que en el caso de la neumonía son producidos por fluido en los pulmones (MedlinePlus, 2017d).

Radiografía de tórax: en esta prueba se obtienen imágenes de la estructura interna del tórax, principalmente enfocadas en el área de los pulmones. Esta prueba es muy eficaz para el diagnóstico de neumonía; sin embargo, no determina el tipo de germen que la causa, aunque se realizan exámenes microbiológicos básicos que permiten la identificación del germen causante de la enfermedad (tales como cultivos de expectoración, hemocultivos y cultivos de líquido pleural) (Jiménez y Calvo, 2005).
Pruebas de sangre: a partir de una muestra de sangre, se realiza un Conteo Sanguíneo Completo (CSC), con el fin de determinar el número de células blancas en la sangre, dado que éstas pueden determinar si existe o no una infección que permita un tratamiento oportuno.

Tomografía computarizada de tórax: es una prueba que permite obtener imágenes precisas de las estructuras del pecho, dando como resultado imágenes más detalladas con respecto a las obtenidas en una radiografía de tórax común.

Oximetría de pulso: dado que la neumonía interrumpe el intercambio de oxígeno en la sangre, con esta prueba se puede estimar la cantidad de oxígeno en la misma, utilizando para ello un sensor de luz colocado en el dedo. A pesar de que las anteriores técnicas tradicionales son eficaces para el análisis in situ, en la literatura existen soluciones alternativas y complementarias para el diagnóstico de la neumonía, que podrían brindar información valiosa para la detección de ésta, incluso en locaciones remotas (Lagor et al, 2001; Abdullah et al, 2011). En este sentido, se pueden encontrar diversos procedimientos modernos orientados a la detección de la enfermedad, que, a partir de datos y señales extraídas del paciente, se podrían conseguir valiosas ayudas para el diagnóstico de la enfermedad. Este tipo de procedimientos se vislumbran como poderosas herramientas que no solo potencializan el diagnóstico in situ, sino que han abierto el camino hacia la posibilidad de un pre-diagnóstico temprano e incluso remoto de la misma (Lagor et al, 2001; Shengjun, Liu, 2010; Abdullah et al, 2011).

Las nuevas herramientas conllevan desde el uso de procedimientos estadísticos, hasta sistemas de inteligencia artificial. A continuación, se realiza una exposición a las diferentes aproximaciones encontradas en la literatura.

\section{TECNOLOGÍAS EMERGENTES}

Una de las primeras formas de complementar el diagnóstico tradicional de la neumonía, se basa en la adquisición, almacenamiento y posterior procesamiento de señales adquiridas por sistemas de auscultación (Barrera, 2016). Este tipo de señales, provienen de la "observación" de una magnitud física en función de variables independientes de tiempo y/o espacio, realizada de tal modo que contenga información valiosa de los 
procesos observados (Alvarado, 2011). En este sentido, el solo hecho de tener una señal digitalizada, permite no solo un eficaz almacenamiento, sino que también abre un amplio abanico de posibilidades de procesamiento; facilitando desde análisis visuales y estadísticos de estas señales, hasta implementaciones de poderosos algoritmos computacionales con multiplicidad de aplicaciones: clasificación de señales, identificación de patrones, modelamientos físicomatemático, etc. Por lo tanto, los sistemas modernos de procesamiento digital de señales han permitido en las últimas décadas, brindar un importante apoyo en la detección de patrones relevantes en las señales adquiridas del cuerpo humano, lo que a su vez ha potencializado la manera en la que se puede realizar el diagnóstico de múltiples enfermedades, facilitando incluso la prescripción del tratamiento (Barrera, 2016; Financial Times, 2017).

Concretamente, en cuanto al diagnóstico de enfermedades pulmonares, los adelantos tecnológicos han permitido el desarrollo de sistemas basados en el análisis computacional de patrones típicos presentes en señales provenientes del sistema respiratorio. Esta identificación de patrones es de gran ayuda al personal médico en la identificación de enfermedades (Gadge et al., 2012).

Adicionalmente, el procesamiento digital de señales también se puede utilizar en la creación de sistemas de clasificación que permitan, mediante técnicas de reconocimiento de patrones, determinar con cierta certeza, la presencia o no de anomalías en los patrones entregados al sistema (OrjuelaCañón y Gómez-Cajas, 2014). De otra parte, mediante el modelamiento físico-matemático de tejidos, es posible diferenciar tejidos sanos de tejidos enfermos, facilitando el diagnóstico de patologías presentes, así como también su posible tratamiento (Correa, 2016). Finalmente, el uso de nuevas tecnologías de telecomunicaciones presentes en dispositivos portables, ha abierto enormes posibilidades a la hora de permitir el desarrollo de herramientas que puedan ser utilizadas de forma remota, facilitando el diagnóstico temprano de enfermedades (MayhuaLopez et al, 2009).

Además de las ventajas ya enunciadas del tratamiento digital de señales, es de destacar que los análisis de laboratorio y el proceso de obtención de radiografías (como la de tórax en el caso de la neumonía), utilizan materiales costosos y contaminantes. Por tanto, este tipo de procedimientos tradicionales resultan difíciles de realizar e interpretar en lugares remotos (rurales o de difícil acceso), sin el acompañamiento de personal calificado y de un espacio adecuadamente acondicionado (Correa, 2016).

Por lo anterior, las primeras aproximaciones que se encuentran en la literatura, abordan el diseño de sistemas de análisis de señales provenientes del cuerpo humano, que, en el caso de la neumonía, pueden ser obtenidas como señales de audio: tos, respiración, habla; o como imágenes: del pulmón, de tejidos (ambos tipos de señales provenientes del sistema respiratorio humano). En el primer caso, un método común para la obtención de las señales acústicas, consiste en el uso de la auscultación (que puede ser digital), a través de la cual se puede realizar el análisis acústico de los pulmones. Posteriormente, la señal adquirida es procesada mediante técnicas de tratamiento digital, para que pueda ser visualizada, almacenada y reproducida en futuros análisis (Barrera, 2016). En este caso, a las señales almacenadas, se les podría aplicar también procedimientos de adecuación, tales como filtrando y eliminación de ruidos $\mathrm{u}$ otras distorsiones (Gadge et al, 2012). Las señales resultantes, servirían de apoyo al personal médico, facilitando el diagnóstico de patologías, la toma de decisiones, o incluso el uso como bases de datos de entrenamiento (RALE, 2008).

Dentro de los primeros estudios, cabe resaltar aquellos que realizan análisis de crackles (sonidos discontinuos de corta duración), muy útiles en el diagnóstico de neumonía y de otras enfermedades respiratorias (Kahya y Güler, 1992; Gadge et al, 2012; Rizal et al, 2016; Grønnesby y Solis, 2017). En este caso, mediante software especialmente diseñado, se pueden procesar diferentes sonidos respiratorios, aplicando técnicas como la Transformada Rápida de Fourier (Fast Fourier Transform - FFT), para su análisis en diversos rangos de frecuencia por medio de filtros; permitiendo determinar, a partir de análisis de potencias pico (Gadge et al, 2012), la presencia de crackles en ciertos rangos de frecuencias causados por enfermedades como bronquitis aguda, asma, neumonía, entre otras (MedlinePlus, 2017d). En este sentido, otros estudios que plantean la detección de crackles, basan su análisis en la determinación de parámetros característicos de éstos, tales como: Anchura Inicial de Deflexión, Duración Total, o Frecuencia de Pico (Kahya y Güler, 1992). De igual manera, utilizando Redes Neuronales Artificiales (o Artificial Neural 
Networks, ANN), también es posible realizar detección de crackles (Kalayci y Çelebi, 1992).

A partir de la identificación de señales con variaciones rápidas (tal como sucede con los crackles) utilizando análisis de Entropía de Tsallis (Tsallis Entropy, TE) en conjunto con ANN, se han logrado alcanzar tasas de acierto superiores al $93 \%$ en su detección (Rizal et al, 2016). También con el uso de Máquinas de Vectores Soporte (Support Vector Machine, SVM) (Betancourt, 2005), han desarrollado estudios para la detección de crackles en sonidos pulmonares obtenidos por auscultación; alcanzando una tasa del $86 \%$ de acierto en la clasificación, utilizando algoritmos, que, de acuerdo a los autores, podrían ser utilizados en dispositivos móviles (Santafé Y E. et al., 2012, Grønnesby y Solis, 2017).

En línea con la detección de crackles, la presencia de sibilancias en la respiración (sonidos agudos similares a un silbido), está asociada a diversas patologías, entre las cuales se cuentan: asma, Enfermedad Pulmonar Obstructiva Crónica $(E P O C)$, infección viral, neumonía, entre otras. De tal manera, que también se encuentran en la literatura, estudios que procuran la detección de éstas en sonidos pulmonares. Concretamente, en ((Parra Ortega C, Herrera Vergara J.2013, Taplidou y Hadjileontiadis, 2010), se realiza un análisis de sibilancias utilizando características espectrales de orden superior wavelet, para evaluar un conjunto de datos adquiridos de pacientes adultos con asma diagnosticada y EPOC. La evaluación estadística del conjunto de características revela la capacidad de discriminación entre las dos patologías, que, de acuerdo a los autores, allanaría el camino para el uso de un clasificador eficiente como mecanismo de discriminación. En este sentido, para alcanzar un sistema de detección automática de sibilancias, a partir del cálculo de espectrogramas, en (Riella et $a l, 2009)$ se utiliza una herramienta de clasificación basada en Redes Neuronales Artificiales. Los resultados expuestos alcanzan una precisión en la tasa de detección de sibilancias de 92\% aproximadamente. Finalmente, utilizando SVM y características de tiempo-frecuencia extraídas de sonidos pulmonares, en (Chamberlain et al, 2015) plantean la identificación de sibilancias que, de acuerdo a los autores, también podrían ser identificadas de forma remota utilizando dispositivos portables.

En otros estudios, se parte directamente del análisis de los sonidos respiratorios en su conjunto, para extraer diferentes tipos de características que permitan realizar una clasificación de personas sanas o enfermas (con una determinada enfermedad). Dichos análisis buscan realizar una detección automática o semi-automática partir de los sonidos capturados del sistema respiratorio. Lo anterior, persigue entre otras cosas, que la detección de una determinada patología se pueda realizar de manera remota, brindando una valiosa ayuda a personas con síntomas de enfermedades como la neumonía, que justamente tiene una alta incidencia en poblaciones rurales, o de difícil acceso y en población vulnerable, de escasos recursos, infantil y de la tercera edad (Ordoñez et al, 2015). Dado lo anterior, en (Yamashita y Himeshima, 2014) se plantea una clasificación robusta para distinguir entre sonidos pulmonares de personas sanas y sonidos anormales. Los sonidos de pulmón utilizados fueron contagiados con sonidos cardiacos, utilizando modelos estocásticos en un sistema clasificatorio basado en Modelos Ocultos de Markov (Hidden Markov Model, $H M M)$. Dicha clasificación alcanzó una tasa de acierto de $83 \%$. Por otra parte, a pesar de que en la literatura existen diversos trabajos que utilizan diferentes procedimientos de clasificación, se destaca el estudio realizado en (Palaniappan et al, 2013), dada la comparación que realiza de varios métodos de clasificación en el mismo estudio. Dicho trabajo utiliza sonidos respiratorios captados de la cavidad torácica abdominal mediante auscultación, procurando evaluar anomalías y trastornos respiratorios. En dicho estudio, utilizan Redes Neuronales Artificiales, Modelos Ocultos de Markov, Modelos de Mezclas de Gaussianas (Gaussian Mixture Models, GMM), Algorítmo K$N N$ (K-Nearest Neighbors), concluyendo que las Redes Neuronales y el Algoritmo K-NN brindan los mejores resultados en cuanto a la monitorización de sonidos respiratorios. De otra parte, existen estudios que utilizan sistemas híbridos de análisis de señales para robustecer el proceso de detección de enfermedades en presencia de otras patologías; ese es el caso del estudio realizado en (Sánchez et al, 2013), que combina un Análisis de Componentes Principales (Principal Component Analysis, PCA) con Redes Neuronales Probabilísticas (Probabilistic Neural Network, $P N N)$, para conseguir una detección de Neumonía Adquirida en Comunidad coexistente con EPOC. Sus resultados alcanzan, mediante validación cruzada, una sensibilidad del $72 \%$, y una especificidad del $81.8 \%$, sugiriendo además una auscultación electrónica realizada en un solo lugar. Los autores sugieren que la herramienta descrita ayudaría en la toma de decisiones en áreas sin instalaciones radiológicas disponibles, y que podría 
tener un gran impacto en aplicaciones de telemedicina. Finalmente, mediante el uso del denominado Modelo Fuente Filtro, una señal puede ser enviada a través de un tejido al que atraviesa, cambiando las características de la señal original. En este escenario, conociendo tanto la señal original, como la señal que ha atravesado el tejido, es posible plantear un modelo físicomatemático que aproxime el comportamiento del tejido para su posterior análisis (Gómez Cajas et al., 2014). En este sentido, también se pueden determinar patologías presentes en tejidos humanos que dan importantes indicios de presencia de determinadas enfermedades. Así, en (Correa, 2016) se utiliza una herramienta basada en ecografías pulmonares utilizando sonidos de muy alta frecuencia, de tal forma que cuando éstos chocan contra los pulmones, mediante la observación de imágenes construidas a partir de los sonidos reflejados, se puede determinar el contenido del pulmón. Dado que los pulmones en condiciones normales están llenos de aire, las imágenes ilustrarán dicho contenido; mientras que, en presencia de neumonía, el contenido aéreo es reemplazado por líquido (consistente de pus, moco, sangre o bacterias), provocando que las imágenes producto de la ecografía resulten muy diferentes a las de un pulmón sano. De esta forma, de acuerdo a los autores, la ecografía pulmonar serviría para distinguir el contenido líquido de una persona con neumonía, del de uno aéreo de un pulmón sano. Un estudio también basado en el uso de ecografías pulmonares, que buscar robustecer la detección de la neumonía es presentado en (Barrientos et al, 2016). Dicho estudio, utiliza un sistema de reconocimiento de patrones, que, mediante un algoritmo de procesamiento digital de imágenes, filtrar ruidos introducidos por la piel, para conseguir una detección computarizada y robusta.

\section{DISCUSIONES Y CONCLUSIONES}

En general, las enfermedades pulmonares causan millones de infecciones a nivel mundial; sin embargo, la neumonía, por tener una muy alta incidencia en poblaciones vulnerables, como niños $\mathrm{y}$ adultos de la tercera edad, ha sido objeto de múltiples estudios para mejorar su detección, especialmente si ésta puede ser temprana, para evitar que llegue a ser fatal. En este sentido, son importantes los avances logrados, pues permiten no solo ayudar al diagnóstico in situ, sino que incluso, de acuerdo a varios autores, sería posible realizar pre-diagnósticos de forma remota, con ayuda de tecnologías crecientes en torno a la telemedicina.
De esta manera, con la ayuda de estas nuevas herramientas y en conjunto con adecuadas políticas estatales, se podría incorporar el uso de estas nuevas tecnologías en los procedimientos estandarizados de diagnóstico. La tendencia de los estudios va en diversos caminos. Sin embargo, la mayoría de ellos utiliza señales audibles como fuente principal de información, bien sea por auscultación o por captura de sonidos producidos por el sistema fonador humano (tos, respiración, voz, etc.). Otra importante tendencia, apunta al uso de imágenes, bien sea a través del uso de ecografías, o a partir imágenes de rayos $\mathrm{X}$ (ecografías de pulmón, o radiografía de tórax).

Por otra parte, en cuanto a las técnicas de análisis de la información, una tendencia apunta al uso de sistemas de reconocimiento de patrones directamente sobre las señales y/o datos adquiridos (ANN, SVM, HMM, etc.); y otra tendencia apunta hacia el análisis de características presentes en las mismas señales (crackles, sibilancias, etc.). Ver Tablas 1, 2 y 3.

Tabla 1. Resumen de técnicas de reconocimiento de patrones utilizadas en la revisión bibliográfica.

\begin{tabular}{l} 
de patrones utilizadas en la revisión bibliográfica. \\
\hline \multicolumn{1}{|c|}{ Técnicas de Clasificación Utilizadas } \\
\hline Redes Neuronales Artificiales \\
\hline Máquinas de Vectores Soporte \\
\hline Algoritmo K-NN \\
\hline Modelos de Mezclas de Gaussianas \\
\hline Modelos Ocultos de Markov \\
\hline
\end{tabular}

Tabla 2. Resumen de tipos de información utilizada para el análisis y/o clasificación. Tipos de información utilizada

Sonidos respiratorios por auscultación Sonidos respiratorios del Sistema Fonador Humano. Imágenes de pulmón de ecografías

Imágenes radiológicas de tórax

Datos clínicos (múltiples tipos de datos)

Tabla 3. Resumen de patrones típicos. Características determinantes analizadas Crackles en sonidos respiratorios Sibilancias en sonidos respiratorios Presencia aire en ecografías de pulmón Presencia líquidos en ecografías de pulmón

Finalmente, existe la posibilidad de incorporar los algoritmos desarrollados en los cada vez más potentes teléfonos inteligentes. Esto conllevaría a una verdadera masificación de este tipo de herramientas, venciendo a su vez una gran cantidad de barreras burocráticas. Sin embargo, la libre distribución de los mismos también podría resultar peligroso para los usuarios; pues si éstos no son 
prudentes, podrían correr graves riesgos en casos en donde la atención médica es indispensable.

No obstante, la incorporación de estas nuevas tecnologías, sería de gran ayuda a la hora de tomar decisiones en un diagnóstico médico, tal como lo afirman diversos autores.

\section{REFERENCIAS}

Abdullah, A., Posdzi, N. et al. (2011). Preliminary study of pneumonia symptoms detection method using Cellular Neural Network. Electrical, Control and Computer Engineering (INECCE), 2011 International Conference on (pp. 497-500).

Álvarez, F., J., Díaz, Baquero, J., F. et al. (2017). Neumonías adquiridas en la comunidad. Unidad Médico-Quirúrgica de Enfermedades Respiratorias (UMQUER).

Alvis, N., De la Hoya, F, Higuera, A. et al. (2014). Costos económicos de las neumonías en niños menores de 2 años de edad en Colombia. Revista Panam Salud Publica. 2005; 17(3):178-83.

Barrera, Sosa, J., A. (2016). Diseño de un sistema de análisis para las señales acústicas pulmonares (Bachelor's thesis, Universidad Militar Nueva Granada).

Barrientos, F. et al. (2016). Filtering of the skin portion on lung ultrasound digital images to facilitate automatic diagnostics of pneumonia. IEEE 36th Central American and Panama Convention.

Beran, D., Zar, H. J. et al. (2015). Burden of asthma and chronic obstructive pulmonary disease and access to essential medicines in low-income and middle-income countries, The Lancet Respiratory Medicine.

Betancourt, G., A. (2005). Las máquinas de soporte vectorial (svms). Scientia et technical, 1 (27).

Calderón, C. y Dennis, R. (2014). Costos económicos de neumonía adquirida en comunidad, meningitis y bacteriemia por Streptococcus pneumoniae en una población adulta que requirió hospitalización en Bogotá, Colombia. Biomédica Revista del Instituto Nacional de Salud, Vol.34, No.1.

Chamberlain, D., Mofor, J. et al. (2015). Mobile stethoscope and signal processing algorithms for pulmonary screening and diagnostics. Global Humanitarian Technology Conference (GHTC).
Cook, P., M., Roger, P., E. et al. (1998) Pathogenesis of pneumovirus infections in mice: detection of pneumonia virus of mice and human respiratory syncytial virus mRNA in lungs of infected mice by in situ hybridization. Journal of general virology 79.10 (1998): 2411-2417.

Correa, M. (2016). ¿Puede la inteligencia artificial salvar la vida de niños con neumonía? TEDx conference.

Fernández, F. y Garro, P. (2015). Neumonía Vírica en el ámbito de la medicina intensiva. Conferencia de Expertos de la SOCMIC.

Gadge, P. B. y Mokal, B. et al. (2012). Respiratory Sound Analysis using MATLAB. International Journal of Scientific AND Engineering Research, 3.

García, Satué, J. y Aspa, Marco, J. (2005). Neumonías. Monografías NEUMOMADRID. Vol. 9.

Gómez-Cajas, D., F., Sepúlveda-Sepúlveda, F., A. et al. (2014). Parametrizaciones robustas de Reconocimiento Automático de Habla (RAH) en redes de comunicaciones. INGE@ UANTendencias en la Ingeniería, 3(6) Vol. 3 • No. $6 \cdot \mathrm{pp}$ 54-64.

Grønnesby, M. y Solis, J. C. A. (2017). Machine Learning Based Crackle Detection in Lung Sounds. arXiv preprint arXiv:1706.00005.

Guzmán, A. y de la Hoz Restrepo, N. (2005). Costos económicos de las neumonías en niños menores de 2 años de edad, en Colombia.

INFAC. (2016). Neumonía adquirida en la comunidad. vol.24, No.05.

INS. (2017a). Enfermedades Transmisibles.

INS. (2017b) ¿Qué son las IAAS?

Kahya, Y., P. y Güler, E. Ç. et al. (1992). Detection and clustering analysis of crackles in respiratory sounds. In Engineering in Medicine and Biology Society, 1992 14th Annual International Conference of the IEEE, Vol. 6, pp. 2527-2528.

Kalayci, T., Çelebi, G. et al. (1992) Automatic detection and classification of crackles by using a neural network. In Engineering in Medicine and Biology Society, 1992 14th Annual International Conference of the IEEE vol. 6, pp. 2580-2581.

Lagor, C., Aronsky, D. et al. (2001). Automatic identification of patients eligible for a pneumonia guideline: comparing the diagnostic accuracy of two decision support 
models. Studies in health technology and informatics, (1), 493-497.

Luna, C., M., Calmaggi, A. et al. (2005). Neumonía adquirida en la comunidad: guía práctica elaborada por un comité intersociedades. Scielo Argentina.

Martínez, C. E., Jaimes, F., A. et al. (2003). Proceso de atención y prescripción en neumonía adquirida en la comunidad en hospitales universitarios en Colombia. Revista Colombiana de Neumología, Vol. 15, No. 4.

Mayhua-lopez, E., ludena-choez, J. et al. (2009). Detección de neumonía a través de la red de telefonía celular.

Ministerio de Salud y Protección Social. (2016), Análisis de situación de salud (ASIS).

OMS. (2016). Neumonía. Centro de prensa, nota descriptiva.

OMS. (2017). Enfermedades No Transmisibles, Organización Mundial de la salud.

Ordoñez, Cedillo, J., Paida, Cuzco, L. et al. (2015). Determinantes sociales que influyen en la neumonía en niños menores de 5 años que acudieron al Subcentro de Salud de Ricaurte en el periodo de enero 2010-diciembre 2013.

Orjuela-Cañón, A., D., Gómez-Cajas, D., F. et al (2014). Artificial neural networks for acoustic lung signals classification. In Iberoamerican Congress on Pattern Recognition (pp. 214221).

PAHO. (2014). Communicable Diseases.

PAHO. (2016). Indicadores Básicos 2016. Situación de la Salud en las Américas, Organización Panamericana de la Salud.

Palaniappan, R. y Sundaraj, K. et al. (2013). Computer-based respiratory sound analysis: a systematic review. IETE Technical Review, 30(3), 248-256.

Parra Ortega C, Herrera Vergara J. (2013). Aplicación de los sistemas de detección de intrusos y la tecnología de agentes en el monitoreo inteligente de redes de datos. Revista Colombiana de Tecnologías de Avanzada ISSN: 1692-7257 - Volumen 2 Número 22.

Pio, A., y Leowski, J. (1984). Programa de la Organización Mundial de la Salud de Infecciones Respiratorias Agudas en la Infancia.

Riella, R., J., Nohama, P. et al. (2009). Method for automatic detection of wheezing in lung sounds. Brazilian Journal of Medical and Biological Research, 42(7), 674-684.

Rizal, A., Hidayat, R. et al. (2016). Pulmonary crackle feature extraction using tsallis entropy for automatic lung sound classification. In Biomedical Engineering (IBIOMED), International Conference on (pp. 1-4). IEEE.

Santafé Y, B D. Chaparro, J Franco (2012). Deteccción de patrones característicos con transformadas wavelet en señales electromiográficas del cuadriceps. Revista Colombiana de Tecnologías de Avanzada ISSN: 1692-7257 - Volumen 2 - Número 20.

Shengjun, L. y Liu, Y. (2010). Feature extraction of lung sounds based on bispectrum analysis. Information Processing (ISIP), Third International Symposium on, pp. 393-397.

Taplidou, S. A. y Hadjileontiadis, L., J. (2010). Analysis of wheezes using wavelet higher order spectral features. IEEE Transactions on biomedical engineering, 57(7), 1596-1610.

UNICEF. (2007). Progreso Para La Infancia.

WHO. (2015) Health Statistics data visualizations dashboard. Noncommunicable diseases and mental health, Global.

Yamashita, M., Himeshima, M. (2014). Robust classification between normal and abnormal lung sounds using adventitious-sound and heart-sound models. In Acoustics, Speech and Signal Processing (ICASSP), 2014 IEEE International Conference on (pp. 4418-4422).

\section{SITIOS WEB}

Geosalud, (2013). Como se diagnostica la neumonía.

http://www.geosalud.com/enfermedades_infe cciosas/neumonia/como-se-diagnostica-laneumonia.html

Allina. (2015). Neumonía Bacteriana. https://www.allinahealth.org/mdex_sp/SD773 7G.HTM

MedlinePlus. (2017a). Sexually Transmitted Diseases.

https://medlineplus.gov/spanish/sexuallytransmitte ddiseases.html

MedlinePlus. (2017b). Mycrobacterial Infections https://medlineplus.gov/spanish/mycobacteria linfections.html

MedlinePlus. (2017c). Neumonía viral. https://medlineplus.gov/spanish/ency/article/0 00073.htm 